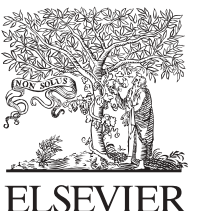

Available online at www.sciencedirect.com

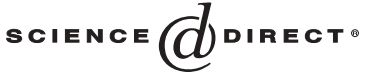

Physica A 342 (2004) 40-47

www.elsevier.com/locate/physa

\title{
Static heterogeneities in liquid water
}

\author{
H. Eugene Stanley ${ }^{\mathrm{a}, *}$, Sergey V. Buldyrev ${ }^{\mathrm{a}, \mathrm{b}}$, \\ Nicolas Giovambattista ${ }^{a}$ \\ ${ }^{a}$ Center for Polymer Studies, Department of Physics, Boston University, Boston, MA 02215, USA \\ ${ }^{\mathrm{b}}$ Department of Physics, Yeshiva University, 500 West 185th Street, New York, NY 10033, USA
}

Available online 2 July 2004

\begin{abstract}
The thermodynamic behavior of water seems to be closely related to static heterogeneities. These static heterogeneities are related to the local structure of water molecules, and when properly characterized, may offer an economical explanation of thermodynamic data. The key feature of liquid water is not so much that the existence of hydrogen bonds, first pointed out by Linus Pauling, but rather the local geometry of the liquid molecules is not spherical or oblong but tetrahedral. In the consideration of static heterogeneities, this local geometry is critical. Recent experiments suggested more than one phase of amorphous solid water, while simulations suggest that one of these phases is metastable with respect to another, so that in fact there are only two stable phases.
\end{abstract}

(c) 2004 Published by Elsevier B.V.

\section{Puzzling behavior of liquid water}

We can superheat water above its boiling temperature and supercool it below its freezing temperature, down to approximately $-40^{\circ} \mathrm{C}$, below which water inevitably crystallizes. In this deeply supercooled region, strange things happen: response functions and transport functions appear as if they might diverge to infinity at a temperature of about $-45^{\circ} \mathrm{C}$. These experiments were pioneered by Voronel and Anisomov in 1971, and developed in detail by Angell and co-workers over the past 30 years [1-4]. Down in the glassy region of water, additional strange things happen, e.g., there is not just one glassy phase [1]. Rather, just as there is more than one polymorph of crystalline water, so also there appears to be more than one polyamorph of glassy water. The first clear indication of this was a discovery of Mishima in 1985: at low

* Corresponding author. Tel.: +1-617-3532617; fax: +1-617-3533783.

E-mail address: hes@bu.edu (H.E. Stanley). 
pressure there is one form, called low-density amorphous (LDA) ice [5], while at high pressure, Mishima discovered a new form called high-density amorphous (HDA) ice [6]. The volume discontinuity separating these two phases is comparable to the volume discontinuity separating low-density and high-density polymorphs of crystalline ice, 25-35\% [7,8]. In 1992, Poole and co-workers hypothesized that the first-order transition line separating two glassy states of water does not terminate when it reaches the no-man's land, but extends into it [9]. If experiments could avoid the no-man's land connecting the supercooled liquid with the glass, then the LDA-HDA first-order transition line would continue into the liquid phase. This first-order liquid-liquid (LL) phase transition line separates two phases of liquid-high-density liquid (HDL) and low-density liquid (LDL) — which are the precise analogs of the two amorphous solids LDA and HDA. Essentially like all first-order transition lines, the LL transition line between noncrystalline phases must terminate at a critical point. Above the critical point is an analytic extension of the LL phase transition line called the Widom line, this extension exhibits apparent singularities-i.e., if the system approaches the Widom line, then thermodynamic response functions appear to diverge to infinity until the system is extremely close, when the functions will round off and ultimately remain finite-as seen in the adiabatic compresibility [10].

\section{Plausibility arguments for static heterogeneities}

That an LL phase transition exists is at least plausible. Plausibility arguments are designed to convince a stranger. My grandmother is not a stranger to me, but she is a stranger to the puzzling behavior of liquid water. One day she asked, "Why do liquids condense? Why do molecules floating around in the air suddenly decide to condense?" To answer, I drew the interaction potential between two water molecules, and explained that there is a minimum at which the water molecules are closer to one another and are more ordered than in the gas-i.e., they have a lower specific volume and a lower entropy. Associated with that minimum is a condensed phase, which we call liquid. My grandmother's follow-up question was right on point: "Why only one minimum?" This was on point because in liquid water there may very well be two minima. This is the case because liquid water is a tetrahedral liquid, and two water tetrahedra can approach each other together in many different ways. One way is coplanar, as in ordinary hexagonal ice $I_{h}$, creating a "static heterogeneity" with a local density not far from that of ordinary ice, about $0.9 \mathrm{~g} / \mathrm{cm}^{3}$. A second way is altogether different: one of the two tetrahedra is rotated $90^{\circ}$, resulting in a closer distance where the minimum of potential energy occurs, and hence a static heterogeneity with a local density substantially larger (by about 30\%) than that of ordinary ice [11]. In fact, this rotated configuration occurs in solid crystalline water ("ice VI"), which occurs at very high pressure. In liquids close to the freezing temperature, there are heterogeneities with local order resembling that of the nearby crystalline phases. Not surprisingly, then, in water at low pressure, there are more heterogeneities that have ice-like order (entropy) and density, while at high pressure there are more heterogeneities that have an ice VI-like order and density. The potential that I drew for my grandmother could not represent all the 
possible relative orientations of two water tetrahedra-rather it simply had two wells: a deeper, "high-volume, low-entropy" well corresponding to the LDL and a shallower, "low-volume, high-entropy" well corresponding to the HDL. Note that the LDL has a higher specific volume and a lower entropy. Therefore-if this double-well potential exists - when water cools, each molecule must decide how to partition itself between these two minima. The specific volume fluctuations increase because of these two possibilities. The entropy fluctuations also increase, and the cross-fluctuations of volume and entropy have a negative contribution, i.e., high volume corresponds to low entropy so that the coefficient of thermal expansion, proportional to these cross-fluctuations, can become negative. The possibility that these static heterogeneities gradually shift their balance between low-density and high-density as pressure increases is plausible, but need not correspond to a genuine phase transition. There is no inherent reason why these heterogeneities need to "condense" into a phase, and the first guess might be that they do not condense-what is now called the singularity free hypothesis $[12,13]$. However if we reason by analogy with the gas-liquid transition, then there is one reason to believe that they will condense. This is related to the fact that a permanent gas is impossible so long as there is a weak attraction, no matter how weak. If such a weak attraction has an energy scale $\varepsilon$, at low enough temperature $T$, the ratio $\varepsilon / T$ will become large enough to influence Boltzmann factor sufficiently that the system will condense. For example, if a single-well potential (e.g., a lattice-gas fluid) with $\varepsilon=1$ condenses below $T_{c}=1$, then if $\varepsilon=0.001$, one anticipates condensation below $T_{c} \approx 0.001$. For this reason, one anticipates that at low enough temperature the one-component liquid would condense into a low-density liquid corresponding to a deeper potential well.

\section{Classic experiments}

The most obvious hallmark of a phase transition line terminating at a critical point is that there occur fluctuations on all length scales up to the size of the correlation length. Since the correlation length is substantial even relatively far from the critical point, one can see manifestations of critical fluctuations in an entire "critical region" extending typically a factor of $\approx 2$ in all thermodynamic directions away from the critical point. For example, extremely close to water's gas-liquid critical point, the correlation length is so large that it becomes comparable to the wavelength of visible light, and one can actually see with the naked eye an eerie glow called critical opalescence, first discovered and correctly interpreted by Andrews in 1869. Since the LL critical point lies well within the no-man's land, it is not possible to see critical opalescence in water. Our experimental evidence for this kind of phase transition must therefore be indirect. Nonetheless, the critical region is sufficiently large to significantly perturb measured functions even outside the no-man's land. For example, Angell and his collaborators have found linear behavior when they plot functions double logarithmically against $T-T_{s}$, where $T_{s}=T_{s}(P)$ is the equation of the Widom line. Typically, the dimensionless temperature variable $\left(T-T_{S}\right) / T_{s}$, does not become smaller than about $7 / 228 \approx 0.03$ [1], due to the presence of the line of homogeneous nucleation temperatures $T_{H}(P)$, which lies just a few degrees above the line of appar- 
ent singularity temperatures $T_{s}(P)$. When we look at experimental data, we find that they are consistent with the possibility of an LL phase transition. The volume fluctuations are proportional to the compressibility and this compressibility is a spectacularly anomalous function. Below $46^{\circ} \mathrm{C}$, the compressibility starts to increase as the temperature is lowered. This phenomenon is no longer counterintuitive if the double-well potential is correct. Similarly, below $35^{\circ} \mathrm{C}$, the entropy fluctuations, which correspond to the specific heat starts to increase. Finally, consider the coefficient of thermal expansion, which is proportional to the product of the entropy and volume fluctuations. This is positive in a typical liquid because, large entropy and large volume go together, but for water this cross-correlation function has a negative contribution and as we lower the temperature this contribution gets larger and larger until we reach $4^{\circ} \mathrm{C}$, at which point the coefficient of thermal expansion passes through zero.

\section{Simulation studies on static heterogeneities}

As the direct experimental study of supercooled water is extremely difficult, several research groups began using computer simulations to look for static heterogeneities. Computer simulations of the microscopic nature of local density fluctuations in water were first done in 1982 [14], but these authors did not identify two distinct static heterogeneities. The prediction of static heterogeneities with two well-defined local volumes is born out by a detailed simulation studies of Errington and Debenedetti, who found two separate peaks (with an "isosbestic point") in the histogram of orientational order-parameter [15]. In contrast, for a simple Lennard-Jones fluid they found a single peak. That the static heterogeneities should condense at sufficiently low temperature is also found in simulations using a wide range of molecular potentials, ranging from "over structured" potentials such as ST2 to "under structured" potentials like SPC/E. Recent work has focused on the latest of all water potentials, Tip5p [16]. Regardless of the potential used, all results seem to be consistent with the LL phase transition hypothesis [2]. The LL phase transition hypothesis does not answer the question "what matters?" i.e., it does not tell us which liquids should exhibit LL phase transitions and which should not. It has been conjectured that it is the local tetrahedral geometry of water that matters, since a tetrahedral local geometry leads to static heterogeneities, which leads to LL phase transition [17]. But then, what about other tetrahedral liquids? If we take silicon, which is another tetrahedral liquid, Sastry and Angell [18] found evidence for a LL phase transition. Similarly, phosphorus [19] and $\mathrm{SiO}_{2}$ [20] share properties with liquid water and have a local tetrahedral geometry and experimental evidence supports the LL phase transition hypothesis. However, not all liquids with local tetrahedral geometry behave like water [21]. And, conversely, some liquids which do not possess locally tetrahedral structure exhibit LL phase transitions (see, e.g., the classic work of Aasland and McMillan [22] and Brazhkin and collaborators [23]). It has been argued that LL phase transitions are associated with liquids possessing a line in the $\mathrm{T}-\mathrm{P}$ phase diagram at which the density achieves a maximum [24-26]. 


\section{Recent experiments}

The experimental work of Angell and collaborators shows apparent singularities when experimental data are extrapolated into the no-man's land. Mishima measured the metastable phase transition lines of ice polymorphs and found that the slopes of these lines exhibited sharp kinks in the vicinity of the hypothesized liquid-liquid phase transition line as predicted by extrapolation [27,28]. The nature of these kinds can be explained if we take into account that a nice polymorph must melt in to a metastable liquid before it can recrystallize into a different polymorph. By the Clausius-Clapeyron relation the slope of that first-order metastable melting line must be equal to the ratio of the entropy change divided by the volume change of the two phases that coexist. In one phase, the coexistence is always the high-pressure polymorph of ice. The other phase, is either HDL or LDL. The volumes and entropies of those two liquids are different, and therefore as the first-order solid-liquid phase transition line crosses the hypothesized LL phase transition line, the slope changes. The Gibbs potential of two phases that coexist along a first-order transition line must be equal. We already know the Gibbs potential of all the polymorphs of ice, so we know, experimentally, the Gibbs potential of the LDL and the HDL. From the Gibbs potential of any substance, one can obtain by differentiation, the volume. Thus, if we know the Gibbs potential as a function of temperature and pressure, we know the volume as a function of temperature and pressure, which is called the equation of state. In this way, Mishima and Stanley were able to find an experimental equation of state for water deep inside the no man's land. This is, of course, not quite the same as actually measuring the densities of two liquids coexisting at the LL phase transition line-as was recently observed in phosphorus [19] - since the Mishima experiments concerned metastable melting lines in which Gibbs potentials of the two phases are not necessarily equal to each other. Very recently, Reichert and collaborators [29] discovered experimentally HDL under conditions outside the no-man's land. They studied thin, quasi-liquid layer between ice $I_{h}$ and solid substrate (amorphous $\mathrm{SiO}_{2}$ ). Using a clever experimental technique at Grenoble, they were able to measure the density and found $1.17 \mathrm{~g} / \mathrm{cm}^{3}$, the density of HDA. It only takes one clear experiment to kill a hypothesis. About two years ago, Loerting and collaborators claimed to find not two glassy waters, but three [30,31]. If true, this would seem to destroy the liquid-liquid phase transition hypothesis because we would no longer have two phases of $\mathrm{H}_{2} \mathrm{O}$. In the temperature-pressure phase diagram there is a first-order transition line separating two phases of amorphous solid water, LDA and HDA. Experimentally, we can press on LDA and transform it into HDA, with the volume dropping by about $30 \%$. This is reversible: when we relieve the pressure, the substance returns to the original LDA with hysteresis. This experiment carried out by Mishima [7] can be replicated by simulations. Loerting and collaborators took the same temperature and pressure as in the Mishima experiments and pressed on the LDA to make HDA. They then heated the HDA at constant pressure, from nitrogen temperatures up to $160 \mathrm{~K}$ and cooled it back. They found that when the HDA was heated from 77 to $160 \mathrm{~K}$, the density increased. When it was cooled back, it did not return to the original density, but continued to increase. The structure, as measured by X-rays, was somewhat different. Loerting and collaborators concluded that 
this was a new form of amorphous solid water, which they called very high-density amorphous (VHDA) solid water. Giovambattista and collaborators repeated Loerting's experiment on a computer [32]. Loerting and collaborators created HDA at $T=77 \mathrm{~K}$, heated it, cooled it back, and found a new structure called VHDA. Giovambattista and collaborators used a computer simulation to replicate exactly what Loerting and collaborators found experimentally. Does this new VHDA form weaken the LL phase transition hypothesis? Giovambattista and collaborators have an alternative explanation. They performed a simulation, one that cannot be done in a laboratory. They took the liquid at high pressure and cooled it so rapidly that it jumped across the no-man's land. Experimentally, liquid water is extremely difficult to cool rapidly at high pressure [28] but, with a computer simulation, this extremely rapid cooling can be done. In fact, most computer simulations are of $\ll 100$ ns duration, a time period not sufficient for crystallization to take place [16,33]. The simulated cooling is continuous and the line extrapolates to the VHDA phase, suggesting that it is the VHDA, not the HDA, that is the stable phase and that VHDA (not HDA) is the glass connected to HDL by isobaric heating/cooling. To confirm this interpretation, they measured the structure pair correlation function $g(r)$ for the two methods of producing VDHA, the first being the method of the Loerting experiment, and the second being the sudden cooling method that can only be employed in simulations. The results were the same. So we conclude that we do not have three phases of glassy water, but still only two. There is the LDA phase at low pressure. At high pressure there is a highly metastable HDA phase that, when heated, crosses over a barrier and becomes VHDA. To confirm this transition, we simply wait. If glass is highly metastable, it will become something else. If we plot density as a function of time in nanoseconds, computer simulations show that HDA's density gradually increases until it approaches VHDA. So, the LL phase transition hypothesis suffered a "near-death experience." It appeared that experiment had detected a new phase of glassy water, but instead they had detected the stable form of the high-density phase, i.e., VHDA.

\section{Discussion}

In summary, in the case of statics of liquid water, the presence of a local tetrahedral geometry leads to two distinct forms of local order ("static heterogeneities") differing in specific volume and entropy, with the specific volume and entropy anticorrelated. This fact gives rise to anomalous fluctuations in compressibility, specific heat and coefficient of thermal expansion. Whether at low enough temperatures these small regions of local order condense in to two separate phases (LDA and HDA) is supported by simulations, but is an open question experimentally. Recent experiments suggested more than one phase of amorphous solid water, while simulations suggest that one of these phases, HDA, is metastable with respect to another, VHDA, so that in fact there are only two stable phases: LDA and VHDA. If there are more than two stable forms of amorphous solid water, then the LL phase transition hypothesis could be amended to require more than two phases of liquid water perhaps a less elegant picture, but a possibility consistent with very recent simulations of Brovchenko et al. [34] and models of Buldyrev and Stanley [35]. 


\section{Acknowledgements}

We thank our collaborators, C.A. Angell, M. Canpolat, P. Debenedetti, G. Franzese, A. Geiger, P. Kumar, E. La Nave, G. Malescio, M. Mazza, O. Mishima, S. Mossa, P.H. Poole, R. Sadr, S. Sastry, A. Scala, F. Sciortino, A. Skibinsky, F.W. Starr, J. Teixeira, and M. Yamada. Also we thank NSF, MIUR Cofin 2002 and Firb and INFM Pra GenFdt for support and Boston University Computation Center for the generous allocation of CPU time.

\section{References}

[1] C.A. Angell, Supercooled water, Ann. Rev. Chem. 34 (1983) 593-630.

[2] P.G. Debenedetti, Supercooled and glassy water, J. Phys.: Condens. Matter 15 (2003) R1669-R1726.

[3] P.G. Debenedetti, H.E. Stanley, The physics of supercooled and glassy water, Phys. Today 56 (6) (2003) 40-46.

[4] O. Mishima, H.E. Stanley, The relationship between liquid, supercooled and glassy water, Nature 396 (1998) 329-335.

[5] P. Brügeller, E. Mayer, Complete vitrification in pure liquid water and dilute aqueous solutions, Nature 288 (1980) 569-571.

[6] O. Mishima, L.D. Calvert, E. Whalley, An apparently first-order transition between two amorphous phases of ice induced by pressure, Nature 314 (1985) 76-78.

[7] O. Mishima, Reversible first-order transition between two $\mathrm{H}_{2} \mathrm{O}$ amorphs at $-0.2 \mathrm{GPa}$ and $135 \mathrm{~K}$, J. Chem. Phys. 100 (1994) 5910-5912.

[8] O. Mishima, Relationship between melting and amorphization of ice, Nature 384 (1996) 546-549.

[9] P.H. Poole, F. Sciortino, U. Essmann, H.E. Stanley, Phase behaviour of metastable water, Nature 360 (1992) 324-328.

[10] E. Trinh, R.E. Apfel, Sound velocity of supercooled water down to $-33^{\circ} \mathrm{C}$ using acoustic levitation, J. Chem. Phys. 72 (1980) 6731-6735.

[11] M. Canpolat, F.W. Starr, M.R. Sadr-Lahijany, A. Scala, O. Mishima, S. Havlin, H.E. Stanley, Local structural heterogeneities in liquid water under pressure, Chem. Phys. Lett. 294 (1998) 9-12.

[12] H.E. Stanley, J. Teixeira, Interpretation of the unusual behavior of $\mathrm{H}_{2} \mathrm{O}$ and $\mathrm{D}_{2} \mathrm{O}$ at low temperatures: tests of a percolation model, J. Chem. Phys. 73 (1980) 3404-3422.

[13] S. Sastry, P. Debenedetti, F. Sciortino, H.E. Stanley, Singularity-free interpretation of the thermodynamics of supercooled water, Phys. Rev. E 53 (1996) 6144-6154.

[14] A. Geiger, H.E. Stanley, Tests of universality of percolation exponents for a 3-dimensional continuum system of interacting waterlike particles, Phys. Rev. Lett. 49 (1982) 1895-1898.

[15] J.R. Errington, P.G. Debenedetti, Relationship between structural order and the anomalies of liquid water, Nature 409 (2001) 318-321.

[16] M. Yamada, S. Mossa, H.E. Stanley, F. Sciortino, Interplay between time-temperature-transformation and the liquid-liquid phase transition in water, Phys. Rev. Lett. 88 (2002) 195701.

[17] H.E. Stanley, S.V. Buldyrev, N. Giovambattista, E. La Nave, A. Scala, F. Sciortino, F.W. Starr, Statistical physics and liquid water: 'what matters'?, Physica A 306 (2002) 230-242.

[18] S. Sastry, C.A. Angell, Liquid-liquid phase transition in supercooled silicon, Nat. Mater. 2 (2003) 739-743.

[19] Y. Katayama, T. Mizutani, W. Utsumi, O. Shimomura, M. Yamakata, K.-I. Funakoshi, A first-order liquid-liquid phase transition in phosphorus, Nature 403 (2000) 170-173.

[20] P.H. Poole, M. Hemmati, C.A. Angell, Comparison of thermodynamic properties of simulated liquid silica and water, Phys. Rev. Lett. 79 (1997) 2281-2284.

[21] C.A. Angell, R.D. Bressel, M. Hemmati, E.J. Sare, J.C. Tucker, Water and its anomalies in perspective: tetrahedral liquids with and without liquid-liquid phase transitions, Phys. Chem. Chem. Phys. 2 (2000) 1559-1566. 
[22] S. Aasland, P.F. McMillan, Density-driven liquid-liquid phase separation in the system $\mathrm{Al}_{2} \mathrm{O}_{3}-\mathrm{Y}_{2} \mathrm{O}_{3}$, Nature 369 (1994) 633-636.

[23] V. Brazhkin. S.V. Buldyrev, V.N. Ryzhov, H.E. Stanley (Eds.), New kinds of phase transitions: transformations in disordered substances, Proceedings of the NATO Advanced Research Workshop, Volga River, Kluwer, Dordrecht, 2002.

[24] F. Sciortino, E. La Nave, P. Tartaglia, Physics of the liquid-liquid critical point, Phys. Rev. Lett. 91 (2003) 155701.

[25] G. Franzese, H.E. Stanley, A theory for discriminating the mechanism responsible for the water density anomaly, Physica A 314 (2002) 508-513.

[26] G. Franzese, G. Malescio, A. Skibinsky, S.V. Buldyrev, H.E. Stanley, Generic mechanism for generating a liquid-liquid phase transition, Nature 409 (2001) 692-695.

[27] O. Mishima, H.E. Stanley, Decompression-induced melting of ice IV and the liquid-liquid transition in water, Nature 392 (1998) 164-168.

[28] O. Mishima, Y. Suzuki, Vitrification of emulsified liquid water under pressure, J. Chem. Phys. 115 (2001) 4199-4202.

[29] S. Engemann, H. Reichert, H. Dosch, J. Bilgram, V. Honkimaki, A. Snigirev, Interfacial melting of ice in contact with $\mathrm{SiO}_{2}$, Phys. Rev. Lett. 92 (2004) 205-701.

[30] T. Loerting, C. Salzmann, I. Kohl, E. Mayer, A. Hallbrucker, A second structural 'state' of high-density amorphous ice at $77 \mathrm{~K}$ and 1 bar, Phys. Chem. Chem. Phys. 3 (2001) 5355.

[31] J.L. Finney, D.T. Bowron, A.K. Soper, T. Loerting, E. Mayer, A. Hallbrucker, Structure of a new dense amorphous ice, Phys. Rev. Lett. 89 (2002) 503-506.

[32] N. Giovambattista, H.E. Stanley, F. Sciortino, Relation between the very high-density and the high-density phases of amorphous solid water, Phys. Rev. Lett. (2004).

[33] M. Matsumoto, S. Saito, I. Ohmine, Molecular dynamics simulation of the ice nucleation and growth process leading to water freezing, Nature 416 (2002) 409-413.

[34] I. Brovchenko, A. Geiger, A. Oleinikova, Multiple liquid-liquid transitions in supercooled water, J. Chem. Phys. 118 (2003) 9473-9476.

[35] S.V. Buldyrev, H.E. Stanley, A system with multiple liquid-liquid critical points, Physica A 330 (2003) 124-129. 